\title{
Neural networks as a tool for unit commitment
}

\section{Rønne-Hansen, Peter; Rønne-Hansen, Jan}

\section{Published in:}

Proceedings of the First International Forum on Applications of Neural Networks to Power Systems

Link to article, DOI:

10.1109/ANN.1991.213465

Publication date:

1991

\section{Document Version}

Publisher's PDF, also known as Version of record

Link back to DTU Orbit

\section{Citation (APA):}

Rønne-Hansen, P., \& Rønne-Hansen, J. (1991). Neural networks as a tool for unit commitment. In Proceedings of the First International Forum on Applications of Neural Networks to Power Systems (pp. 266-270). IEEE. https://doi.org/10.1109/ANN.1991.213465

\section{General rights}

Copyright and moral rights for the publications made accessible in the public portal are retained by the authors and/or other copyright owners and it is a condition of accessing publications that users recognise and abide by the legal requirements associated with these rights.

- Users may download and print one copy of any publication from the public portal for the purpose of private study or research.

- You may not further distribute the material or use it for any profit-making activity or commercial gain

- You may freely distribute the URL identifying the publication in the public portal

If you believe that this document breaches copyright please contact us providing details, and we will remove access to the work immediately and investigate your claim 
M.Sc.E.E. Peter Rønne-Hansen and Professor Ph.D. Jan Rønne-Hansen

Electric Power Engineering Dept. Technical University of Denmark DK-2800 Lyngby

\section{Abstract}

In this paper some of the fundamental problems when solving the unit commitment problem by means of neural networks have been attacked. It has been demonstrated for a small example that neural networks might be a viable alternative. Some of the major problems solved in this initiating phase could form the fundament for the analysis of real life sized problems. These will be investigated in the near future.

\section{Introduction.}

Neural networks have for some time proven to be a useful tool when dealing with signal analysis; alarm treatment and certain types of forecasting. In all these applications data can be characterized by an inherent structure clearly suited for pattern recognition.

An investigation of the unit commitment procedure does not reveal an obvious structure even though the skilled operator (expert) is able to make decisions very close to optimum without any analytical tool at hand. It was decided in a masters thesis (total duration 5 months) to make an effort in applying neural networks to this problem area. Some of the findings during this research is described in this paper.

Among the problems which should be solved was of course how to present the problem to the neural network - and thus influencing the construction of the network. Even more difficult was the problem how to describe the load curve in a simple and accurate way.

The knowledge gained is that a structure does exist, however weak and still not obvious from load and unit data. This points to further investigations including problems of real life size. These investigations can - to our opinion - be carried out directly based on the results reported here.

\section{General Description of the} Unit commitment Problem.

In order to consider this work in the perspective of the very large width of the optimization problem, which the unit commitment problem (UC) represents, a general overview of the problem will be given here in short.

As it is seen, the optimization of the power production have to be done, observing a variety of conditions due to as well the system as the individual unit. For the system, the following conditions/contstraints has to be fulfilled:
1.1) Electric power load

1.2) Heat load (when combined production must be taken into account)

1.3) Import/export agreements/contracts

1.4) Spinning reserve requirements

1.5) Fuel supply constraints

When considering the individual unit, the following conditions must be taken into account:

2.1) Production boundaries

2.2) Fuel consumption

2.3) Start/stop costs

2.4) Hot-standby costs

2.5) Scheduled outage

2.6) Specified production/Specific production requirements

2.7) Scheduled commitment

2.8) Maximum step response

2.9) Crew constraints

2.10) Interdependence between heat and power production

2.11) Maximum load gradient

2.12) Minimum up-time

2.13) Minimum down-time

2.14) Penalty factors for network losses

In this rather traditional survey several problems are hidden. The start/stop costs ought to include the capitalization of the increased risk of outages during start up and shut down. These data normally are not available although investigations have shown that such indirect start costs may exceed the direct start costs by several hundred per cent! Neither the fact that additional regulation costs when increasing the power production, to a certain extent can be regained when reducing the production later on are considered. These examples are just two of several.

In the following the problem will be considered as including the traditional constraints only (i.e. $1.2 ; 1.3$ and $2.5-2.11$ are left out).

Even in this case the solution is difficult. Most often less accurate methods are chosen since the complexity of the problem makes the very accurate solution impossible taking into consideration the state of the art of computing as well as lack of data.

\section{Preparing data.}

To get started, using neural networks to the UC problem, it was chosen here to use a sufficiently exact method for a rather small system when producing learning and test data. Hereby the results obtained will be as close 91TH0374-9/91/0000-0266\$01.0001991 IEEE 
to optimum as possible, and the discussion will be concentrated on the application of the neural networks and the experience gained.

Because no program for the solution of the UC problem was at hand it was chosen to use dynamic programming carried out by hand for a sufficiently small example system. When taking into consideration the "normal" expenses (as dealt with in the standard UC problem) the forward dynamic programming procedure gives an accurate answer to the problem. Thus still more simple cases can be defined by neglecting many constraints. The applicability of neural networks for the solution of this problem can be tested beginning with a very simple case and step by step taking into account still more complex examples by adding more constraints from the list above.

A system, which is used in T.S. Dillon's lecture notes [2]. This small 5 unit system has been slightly modified (table 1 ) and used as described in the following. These 5 units give a total of 32 possible commitments, which is too large a number to be dealt with in dynamic programming by hand. These 32 commitments furthermore have been reduced to 22 legal commitments, by neglecting in total $10 \mathrm{com}-$ mitments rejected according to the following rules.

- No units committed (uninteresting)

- only one unit committed (no reserve in case of outages)

- Commitments in which the technical minimum production of the largest unit committed is bigger than the sum of the rated power of all the remaining committed units

\begin{tabular}{|c|c|c|c|c|c|}
\hline UNIT & LEV & $P_{\min / \max }$ & \multicolumn{3}{|c|}{ COSTS } \\
\cline { 4 - 6 } & & MW & base & incremental & startup \\
\hline \multirow{3}{*}{1} & 1 & $80-95$ & & 0.29 & \\
& 2 & $95-135$ & 27.92 & 0.31 & 50 \\
& 3 & $135-150$ & & 0.33 & \\
\hline \multirow{3}{*}{2} & 1 & $40-60$ & & 0.33 & \\
& 2 & $60-92$ & 17.50 & 0.38 & 30 \\
& 3 & $92-100$ & & 0.55 & \\
\hline \multirow{3}{*}{3} & 1 & $20-30$ & & 0.371 & \\
& 2 & $30-40$ & 8.54 & 0.381 & 10 \\
& 3 & $40-50$ & & 0.489 & \\
\hline & 1 & $10-30$ & & 0.45 & \\
& 2 & $30-40$ & 8.25 & 0.465 & 13 \\
& 3 & $40-50$ & & 0.549 & \\
\hline \multirow{3}{*}{5} & 1 & $0-5$ & & 0.40 & \\
& 2 & $5-15$ & 2.00 & 0.40 & 5 \\
& 3 & $15-30$ & & 0.50 & \\
\hline
\end{tabular}

Table I Unit data

When using a discrete dynamic programming method, it is necessary to select a step size, valid for the load as well as for the production.
Having an installed capacity of $380 \mathrm{MW}$, a step size equal to $10 \mathrm{MW}$ was found suitable. For each load step between $50 \mathrm{MW}$ and $380 \mathrm{MW}$, the load dispatch now can be found by using the merit order approach. The application of the simple merit order approach is possible due to the economic structure of the units. However, since the actual load dispatch is solved independently of the unit commitment problem no implication on the function of the neural network will exist. This of course is done for every legal commitment within the actual load step.

Considering the load curves, the following decisions were made. Four load curves from the Danish power system were selected, representing workday and weekend both summer and winter [6]. Furthermore a load curve from CIGRE [1] was selected, and finally a fictitious load curve was constructed. The fictitious load curve was chosen closer to the CIGRE-curve than to the more uniform curves for the Danish power system in order to extend training data for more distinct load variations. All the curves were adjusted to suitable load sizes. Finally each load curve was considered following and followed by an identical load curve (fig. 1).

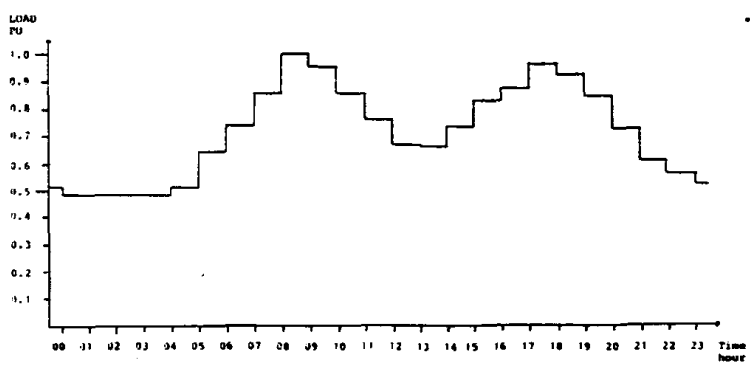

Loadcurve, CIGRE

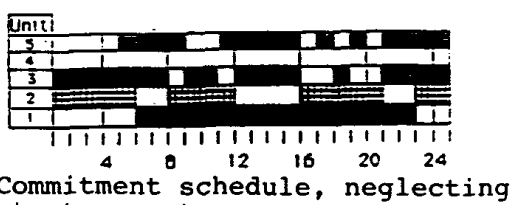
startup costs

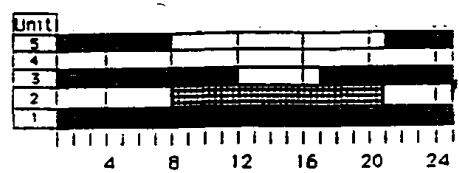

Commitment schedule, including startup costs

Figure 1: Example of a loadcurve with according commitment schedules.

The next point to be handled, is to select a sufficiently simple subset of constraints, by removing most of the items of the original curves. As said, the ldea was to begin as simple as possible, and then extend the complexity,
as the work proceeded. The most simple problem

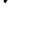


was selected by considering the following constraints only:

$$
\begin{aligned}
& \text { 1.1) Electric load } \\
& \text { 2.1) Production limits } \\
& \text { 2.2) Production costs }
\end{aligned}
$$

The next constraint, which should be considered in addition to the three mentioned is 2.3): Fixed start up costs.

\section{Selecting the neural network. 4.1 Choice of tools}

It does not require a lot of experience with neural networks to observe, that the unit commitment problem is not suited for an auto-associative neural network paradigm but a hetero-associative paradigm must be used. Most obvious then is to use the back propagation paradigm. This because it is the most common used paradigm based on well-known and generally accepted engineering methods. Furthermore the problem is of a continuous character, which excludes e.g. the madaline paradigm.

Two software packages were used. The first one was: NW Explorer from Neural Ware. The application of this is of very basic character and therefore can be considered less practical, however, of great pedagogical importance since the network can be designed directly and the learning process closely followed. The second package was Brainmaker Professional from California scientific Software. The main part of the calculations were carried through using this tool.

\subsection{Designing the network}

Next it was decided, how the output should be represented. In general three obvious possibilities can be chosen:

1) One output neuron/unit $\quad \Rightarrow 5$ outputs

2) One output/possible commitment

3) One output i.e. one neural net/unit

2) is not suitable, because an expansion with one unit, increases the possible commitments with a factor of two. Regarding 3 ) it necessary gets the same fate as 2 ). The reason is that the state of any other unit influences the actual unit, when trying to train the neural network. Neglecting the other units in this phase, makes it much harder, maybe even impossible to make the neural net learning. So the only possible solution left is 1).

\section{Training and testing the networks.}

5.1. The UC procedure neglecting start costs.

In order to begin with what was believed to be the most simple problem even the start costs were neglected. As our knowledge of neural network prior to this investigation was extremely limited we did not realize until much time was spent in vain that this problem was not suited for neural network solution at all, since no structure exists between the solution in one hour and the future as well as the previous periods. At least not when using the traditional data only. Considering such costs as caused by changing the produced power by the single unit some slight connections over time could have been provided, but such data are far less important than start costs and not available in general.

When reflecting the nature of unit commitment without start-up cost, i.e. the solution is found based on the load alone, the question comes up: Why at all, try to solve this problem using neural networks? The reason is, that in general, the problem is solved as a simple table look up, unless there is more than one single solution for each load step. That is exactly the problem here. In a couple of cases two or three equal solutions exist, i.e. different commitments giving the same cost. In this case it makes sense to use a neural net. The aim is to learn a neural net to give the correct answer, without having to care about whether the solution actually is a "single" or one out of several possible.

In order to create some meaningful interaction it was decided to achieve a minimum number of starts when more than one solution to a given problem exist. Despite this criteria a unique solution in a few cases did not exist. Data for the period $(t-1)$ hours to $(t+3)$ hours were used. The learning data were formed by 5 out of the 10 days (each 24 hours) available. The range of power considered was 50-340 MW in steps of 10 MW (as mentioned above).

Several neural network of widely different structure were tested. As common characteristics the output was chosen corresponding to the plants in question (i.e. 5 output neurons) whereas the number of input neurons corresponded to the number of time-units considered in the single case (between 3 and 8 as each hour and its "surrounding" hours form one case). The neural networks were arranged in 1-3 hidden layers and with various couplings between the different layers. The number of neurons in the hidden layers varied between 10 (in a single layer) and 29 (in three layers).

As already mentioned the experience from this part of the investigation was negative and is described here in the hope, that someone else hereby could be prevented from a simitar disappointment. Anyway this is a clear example showing the difference between conventional reasoning when using algorithmic procedures and dealing with learning as applied to neural networks.

\subsection{Including start costs.}

When the start-up costs were taken into account, the UC problem changes its character, compared with the problem described above. The output still is the same. Now the search is headed for a number of inputs, representing the past as well as the present and the future. The aim is, 268 
describe a general structure of the unit commitment problem. The following considerations were made:

The past: Having an actual electric load to be met, the load curve in the past is known as well as the proceeding commitments. It is now proclaimed that all useful information for the past is the proceeding commitments. The reason is that the actual commitment is the chosen solution for the UC problem till now, e.g. it represents all the necessary information of the past.

The present: It is chosen here to partition the load in four intervals: $50-150 \mathrm{~kW}$; 160-250 MW; 260-300 MW and 310-380 $\mathrm{kW}$. The choice of these limits is made by squinting to the minimum and maximum capacities of the different possible commitments. This makes it easier to find the structure, because different neurons become active for different loads. If e.g. the load is in interval 3 , then some of the commitments are illegal!

The future: An option could be to represent the future load in the same way as the present load for 15-20 hours a head. This implicates, however, up to 80 input data. But since the clearness of the problem suffers heavily from such a large number of inputs, it was chosen to describe the future by means of maximum and mean power demand in a few different future time hbrizons. Hereby information about future energy consumption is available and from this it can be decided whether or not the savings by decommiting a unit exceeds the start-up costs or not. If the maximum load in the future, with the same time horizons, are introduced, then it is possible to judge whether or not an actual decommitment is desirable within the actual time horizon. Iet's take an example: if the mean load is low in the period considered but in the middle of the period a load maximum exists which exceeds the maximum power of the commitment considered, it is not advisable to shot down a unit as it must be started up again in the middle of the period.

The mean and maximum values are found for the intervals $2,5,10$ and 15 hours ahead plus the mean value for 20 hours ahead. It was decided to represent the future mean and maximum loads as deviations from the actual load. Hereby it is easier to distinguish between decreasing and increasing load shape. So it is obvious from the sign whether the already committed capacity is sufficient or if shortage/surplus must be taken care of by commiting/decomiting additional capacity.

- 27 inputs now remain in stead of 80 .

A short description of training and test data is necessary too. A total of 17 days were available for the construction of the training data and another six days were selected as test data.

Naturally the start costs will decrease the number of changes in commitment and the learning data when taken chronologically and with the same weight given to every hour will provide very poor training in cases where a change in comitment is necessary. To circumvent this draw-back the learning data are partitioned in three groups, the two first of which contain the "interesting" cases:

- All time steps where a change in commitment is optimal.

- All time steps where a change in commitment could be expected but must not appear.

The third group does not necessarily contain all the remaining data but a selection which ensures the representation of all load sizes in periods of ascending, constant and descending load shape.

Using these criteria 178 time steps were selected as training data from a total of 408 possible time steps.

The test data include the remaining time periods from the 17 days partially included in the learning data (in total 230 time steps remain) plus all the 144 time steps in the 6 days selected for test purposes.

The NN used was a standard back propagation network with 50 neurons in the single hidden layer. Then learning, a noise of 0.05 was introduced. Furthermore a learning coefficient of 0.6 was used, instead of the default value of 1.0. This "deceleration" factor indicates the vague structure of the problen along the time axis.

The mafor experience from the work (table 2) is that the structure seems to exist even though it is rather vague.

\begin{tabular}{|l|c|}
\hline \multicolumn{1}{|c|}{ Test data } & $\begin{array}{c}\text { failure rate } \\
\text { pct. }\end{array}$ \\
\hline Training data & 1.7 \\
\hline $\begin{array}{l}\text { Training days, before selection } \\
\text { of training data }\end{array}$ & 2.7 \\
\hline Total amount of data & 4.5 \\
\hline $\begin{array}{l}\text { Test days without selections } \\
\text { for training }\end{array}$ & 9.7 \\
\hline
\end{tabular}

Table 2: Failure rate for trained network

\section{Conclusion.}

Now let's sum up, what has actually been done, and the results of this.

The UC has been solved optimally for a reduced system. When neglecting start up costs, the structure of the problem was not suitable for solving by $\mathrm{NN}$.

Concerning UC when taking fixed start-up costs into consideration. It was found that some kind of structure can be recognized. Though some important circumstances are observed. 
1. The ability of learning is dependent on the input and output selected.

2. The training data must be selected carefully.

3. With the proposed solutions for the above-mentioned points, the structure is still very vague, which means that

- a low learning coefficient has to be used.

The encouraging fact is, that it has been possible to find a structure for an optimally solved UC even though a simple case only has been used. Thus some weaknesses are present and should be mentioned here.

First, some of the misfits are characterized by illegal commitments e.g. the total capacity of the chosen commitment was insufficient or it belonged to one of the categories previously rejected (as stated above).

secondly, the tests were not carried out where a previously faulty commitment was used as input in the succeeding time step. In other words secondary failures do not exist.

This leads to the question: what next? On the basis of this work it is not possible to judge categorically whether the neural network has a future in this field or not. A lot of work remains. It must be investigated if parameters as e.g. shape of load curve or unit data seriously influence the ability to learn. Further investigations extending training and test data has to be done. Concerning the faulty commitments it seems necessary to design some kind of "shell" surrounding the neural network. This shell prepares the input to each time step e.g. transfers the commitment from the previous time step and prepares the load data for the next 20 hours as described above. Furthermore an analysis of the output for each time step must be carried out by the shell, even though a complete test hardly can be done. The aim will be to obtain a very fast system which always gives feasible solutions and only in rare occasions presents a non-optimal solution, judged from the quality of the learning data, off course. If it is possible for the shall to catch the wrong answers in a relatively simple way it is clear that the secondary faults drop out.

\section{References}

[1] Electra No 39 March 1975 M. Magnien. Report on the optimisation of power system operation.

[2] T.S.Dillon, Lecture note The generator unit commitment problem.

[3] IEEE TPS Vol PWRS 2 No 2 May 1987 w.L.Snyden, H.D.Powell, J.C.Rayburn Dynamoc programming approach to unit commitment.

[4] Neural Ware, Inc. 1989 J.Guiver, G.Pelton m.fl. NW Explorer
[5] California scientific Software 1990, Brainmaker. Neural network simulation software. User's guide and reference manual.

[6] Annual Statistics in Danish Power supply 1989 (in Danish). 